\title{
Structure and Stability of Charged Colloid-Nanoparticle Mixtures
}

\author{
Braden M. Weight and Alan R. Denton* \\ Department of Physics, North Dakota State University, Fargo, ND, U.S.A. 58108-6050
}

(Dated: March 13, 2018)

\begin{abstract}
Physical properties of colloidal materials can be modified by addition of nanoparticles. Within a model of like-charged mixtures of particles governed by effective electrostatic interactions, we explore the influence of charged nanoparticles on the structure and thermodynamic phase stability of charge-stabilized colloidal suspensions. Focusing on salt-free mixtures of particles of high size and charge asymmetry, interacting via repulsive Yukawa effective pair potentials, we perform molecular dynamics simulations and compute radial distribution functions and static structure factors. Analysis of these structural properties indicates that increasing the charge and concentration of nanoparticles progressively weakens correlations between charged colloids. We show that addition of charged nanoparticles to a suspension of like-charged colloids can induce a colloidal crystal to melt and can facilitate aggregation of a fluid suspension due to attractive van der Waals interactions. We attribute the destabilizing influence of charged nanoparticles to enhanced screening of electrostatic interactions, which weakens repulsion between charged colloids. This interpretation is consistent with recent predictions of an effective interaction theory of charged colloid-nanoparticle mixtures.
\end{abstract}

\section{INTRODUCTION}

Suspensions of colloidal (nm- $\mu$ m-sized) particles, dispersed in a solvent by Brownian motion, are characterized by extreme sensitivity of thermal and mechanical properties to environmental conditions [1]. Relatively small changes in internal composition or external fields can modify interparticle interactions and profoundly affect thermodynamic stability and phase behavior of these soft materials. Such diversity and tunability of materials properties makes colloidal suspensions appealing for many practical applications, e.g., in the food, pharmaceutical, and consumer products industries [2, 3].

Opposing thermodynamic stability of colloidal suspensions are attractive van der Waals interparticle forces [4], which drive particles to aggregate and can lead to coagulation and sedimentation. Colloidal particles can be stabilized against aggregation by introducing either steric forces, e.g., by adsorbing or grafting polymers or surfactants to their surfaces, or electrostatic forces, by creating surface charges via dissociation of counterions. Steric and charge stabilization are two established mechanisms known to prevent aggregation [1-5].

Addition of nanoparticles can also affect structure and thermodynamic stability of colloidal suspensions. In a series of experiments, Lewis et al. [6-10] observed that addition of charged zirconia or polystyrene nanoparticles to aqueous suspensions of weakly charged silica colloidal microspheres affected the tendency of the colloidal particles to aggregate. With increasing nanoparticle concentration, suspensions transitioned from unstable to stable and back to unstable. Lewis et al. postulated that nanoparticles may inhibit aggregation by forming halos around the colloidal particles. Numerous independent experimental studies [11-21] have confirmed that nanopar-

\footnotetext{
* alan.denton@ndsu.edu
}

ticle haloing stabilizes mixtures of weakly charged colloids and strongly charged nanoparticles that can adsorb to the colloidal surfaces. At intermediate concentrations, adsorbed nanoparticles increase the magnitude of the zeta potential of the colloids, promoting charge stabilization, while at higher concentrations, nanoparticles screen repulsive electrostatic interactions, destabilizing the suspension. The role of nanoparticles in screening electrostatic interactions is not, however, well understood.

Charged colloidal mixtures have been modeled by a variety of theoretical and computational methods, including computer simulations [22-26], integral-equation theory [27-36], and Poisson-Boltzmann theory [37-41]. Several recent studies of highly asymmetric mixtures of charged colloids and nanoparticles [23, 32, 33, 40, 41] yielded effective colloid-colloid pair interactions that support the postulated nanoparticle haloing mechanism for certain system parameters. However, while previous studies have focused on weakly charged colloids mixed with oppositely-charged nanoparticles, relatively little is known about the influence on suspensions of strongly charged colloids of like-charged nanoparticles.

Motivated by previous experimental observations and modeling results, we conducted a computer simulation study to explore the impacts of nanoparticle doping on the structure and phase stability of charge-stabilized colloidal suspensions. Our study is distinguished from previous work by focusing on mixtures in which both particle species carry significant charge of the same sign. To highlight the influence of electrostatic interparticle interactions, we adopt a practical coarse-grained model in which charged colloids and nanoparticles interact via physically consistent effective pair potentials.

In Sec. II, we define our model, which is derived from the primitive model of charged colloid-nanoparticle mixtures by averaging over microion degrees of freedom. In Sec. III, we outline our methods of molecular dynamics simulation and analysis of structural properties. Section IV presents numerical results for radial distribu- 
tion functions and static structure factors of model saltfree mixtures over ranges of system parameters, including nanoparticle charge and concentration. From these results, we identify general trends in the influence of nanoparticles on pair correlations between charged colloids and on the structure and phase stability of bulk suspensions. Finally, in Sec. V, we summarize and conclude with an outlook for future work.

\section{COARSE-GRAINED MODEL OF COLLOID-NANOPARTICLE MIXTURES}

We consider a bidisperse mixture of $N_{c}$ colloidal particles and $N_{n}$ nanoparticles, of average number densities $n_{c}=N_{c} / V$ and $n_{n}=N_{n} / V$, dispersed in a solvent in a volume $V$ at absolute temperature $T$, along with $N_{\mu}$ microions: $N_{+}$counterions and $N_{-}$coions (see Fig. 1). The colloids and nanoparticles are modeled as charged hard spheres (macroions), of respective radii $a_{c}$ and $a_{n}$ and valences $Z_{c}$ and $Z_{n}$, and the microions as point charges of valences $\pm z$. For simplicity, we consider a symmetric electrolyte of salt ion pairs with the same valence as the counterions. The model is easily generalized, however, to describe asymmetric electrolytes and finite-sized microions. The surface charges of the colloids and nanoparticles originate from dissociation of counterions. Assuming like-charged (negative) particles, global electroneutrality dictates that

$$
Z_{c} N_{c}+Z_{n} N_{n}=z\left(N_{+}-N_{-}\right) .
$$

As an underlying explicit model, we take the primitive model of charged colloidal mixtures, in which the solvent is idealized as a continuum medium of dielectric constant $\epsilon$. Across this effective medium, the van der Waals interaction between particles $i$ and $j$, of radii $a_{i}$ and $a_{j}$, at separation $r$ between the particle centers, is approximated within the Hamaker theory [4] by an effective pair potential of the form

$$
\begin{aligned}
v_{\mathrm{vdW}}(r) & =-\frac{A_{i j}}{6}\left[\frac{2 a_{i} a_{j}}{r^{2}-\left(a_{i}+a_{j}\right)^{2}}+\frac{2 a_{i} a_{j}}{r^{2}-\left(a_{i}-a_{j}\right)^{2}}\right. \\
& \left.+\ln \left(\frac{r^{2}-\left(a_{i}+a_{j}\right)^{2}}{r^{2}-\left(a_{i}-a_{j}\right)^{2}}\right)\right], \quad r>a_{i}+a_{j},
\end{aligned}
$$

where the Hamaker constant $A_{i j}$ depends on the dielectric constants of both the particles and the medium. Since we restrict attention here to like-charged particles whose repulsive electrostatic interactions are sufficiently strong to prevent nanoparticle adsorption, we neglect dielectric polarization effects, as in previous studies of charged colloidal mixtures [23-35, 37-41]. Although induced polarization charges may affect self-assembly of strongly coupled, oppositely charged colloidal mixtures [42], charge-charge interactions are expected to dominate in the moderately coupled, like-charged systems investigated here. Furthermore, our simulations are for the most part limited to system parameters for

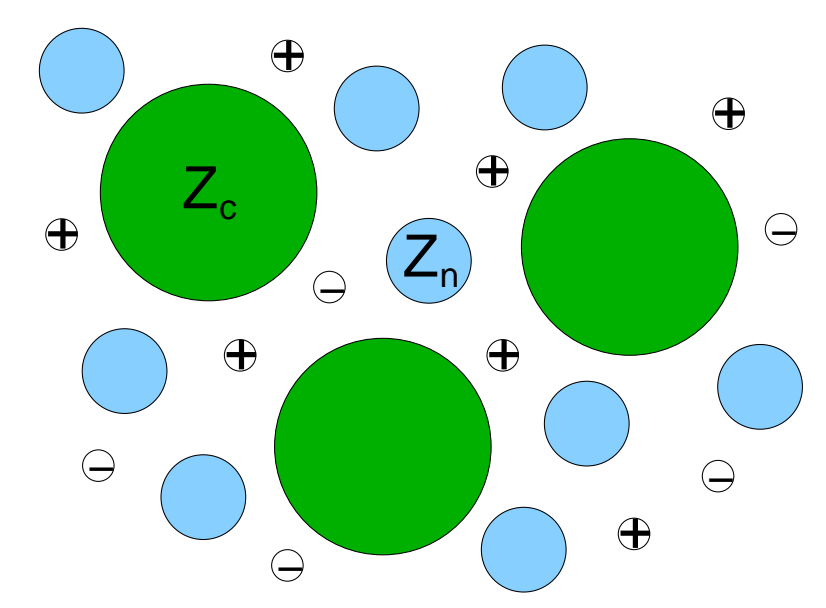

FIG. 1. Primitive model of colloid-nanoparticle mixture: charged colloids (valence $Z_{c}$ ), nanoparticles (valence $Z_{n}$ ), and microions (counterions and coions) in an implicit solvent.

which, as shown below, electrostatic interactions dominate over van der Waals interactions. The systems modeled here thus differ importantly from experimental systems in which van der Waals attraction between nanoparticles and weakly or oppositely charged colloidal particles leads to nanoparticle adsorption and haloing [6-10].

Within the primitive model, all charged particles interact via electrostatic (Coulomb) pair potentials. The model system is formally governed by a Hamiltonian, $H=H_{0}+H_{\text {el }}$, which naturally separates into a reference term $H_{0}$, including the total kinetic energy and particle hard-core interactions, and a term $H_{\mathrm{el}}$ that represents the total electrostatic energy:

$$
H_{\mathrm{el}}=H_{m m}+H_{\mu \mu}+H_{m \mu} .
$$

The three terms on the right side of Eq. (3) account for pair interactions among colloidal and nanoparticle macroions $(m)$ and microions $(\mu)$. These terms can be expressed more explicitly as

$$
\begin{gathered}
H_{m m}=\sum_{i<j=1}^{N_{c}} v_{c c}\left(r_{i j}\right)+\sum_{i<j=1}^{N_{n}} v_{n n}\left(r_{i j}\right)+\sum_{i=1}^{N_{c}} \sum_{j=1}^{N_{n}} v_{c n}\left(r_{i j}\right) \\
H_{\mu \mu}=\sum_{i<j=1}^{N_{+}} v_{++}\left(r_{i j}\right)+\sum_{i<j=1}^{N_{-}} v_{--}\left(r_{i j}\right)+\sum_{i=1}^{N_{+}} \sum_{j=1}^{N_{-}} v_{+-}\left(r_{i j}\right) \\
H_{m \mu}=\sum_{i=1}^{N_{c}} \sum_{j=1}^{N_{\mu}} v_{c \mu}\left(r_{i j}\right)+\sum_{i=1}^{N_{n}} \sum_{j=1}^{N_{\mu}} v_{n \mu}\left(r_{i j}\right)
\end{gathered}
$$

where $N_{\alpha}$ denotes the number of particles of species $\alpha$ and $v_{\alpha \beta}\left(r_{i j}\right)$ is the Coulomb pair potential between particle $i$ of species $\alpha$ and particle $j$ of species $\beta$ separated by center-to-center distance $r_{i j}$. 
The primitive model of charged colloidal mixtures [Eqs. (4)-(6)], wherein all particles interact via longrange Coulomb potentials, poses considerable challenges for computational modeling. Recently, Chung and Denton [43] developed a general effective interaction theory for polydisperse suspensions of charged colloids. Extending to mixtures an established coarse-graining approach [44-47], which traces out microion degrees of freedom from the partition function, the theory maps the multicomponent macroion-microion mixture onto a system of only pseudo-macroions governed by effective electrostatic interactions. Within a linear approximation for the response of microion densities to macroion potentials and a random phase approximation for microion response functions, the colloidal and nanoparticle macroions interact via repulsive hard-core Yukawa (screened-Coulomb) effective pair potentials. In thermal $\left(k_{B} T\right)$ units, the effective electrostatic pair potentials take the form

$$
v_{\mathrm{Y}}(r)=Z_{i} Z_{j} \lambda_{B} \frac{e^{\kappa\left(a_{i}+a_{j}\right)}}{\left(1+\kappa a_{i}\right)\left(1+\kappa a_{j}\right)} \frac{e^{-\kappa r}}{r}, \quad r>a_{i}+a_{j},
$$

where

$$
\kappa=\sqrt{\frac{4 \pi z^{2} \lambda_{B}\left(\left|Z_{c}\right| n_{c}+\left|Z_{n}\right| n_{n}+2 n_{s}\right)}{1-\phi}}
$$

is the Debye screening constant, $\lambda_{B}=e^{2} /\left(\epsilon k_{B} T\right)$ is the Bjerrum length, $k_{B}$ is the Boltzmann constant, and $\phi=(4 \pi / 3)\left(n_{c} a_{c}^{3}+n_{n} a_{n}^{3}\right)$ is the fraction of volume from which the microions are excluded by the particle hard cores. Similar effective pair potentials have been derived previously by integral-equation methods [30] and adopted in simulation studies [48-50], albeit neglecting the excluded-volume factor of $1 /(1-\phi)$ in the screening constant, which can be significant in concentrated suspensions. The effective interaction theory also predicts a one-body volume energy $E_{0}$, which accounts for the microion entropy and macroion-microion interaction energy [43]. While not affecting structural properties, the volume energy influences thermodynamic properties through its dependence on average macroion densities, which can be especially significant in deionized suspensions. The coarse-grained model is thus governed by an effective Hamiltonian, $H_{\mathrm{eff}}=H_{0}+E_{0}+H_{\mathrm{el} \text {,eff }}$, where the last term,

$$
\begin{aligned}
H_{\mathrm{el}, \mathrm{eff}} & =\sum_{i<j=1}^{N_{c}} v_{c c, \mathrm{Y}}\left(r_{i j}\right)+\sum_{i<j=1}^{N_{n}} v_{n n, \mathrm{Y}}\left(r_{i j}\right) \\
& +\sum_{i=1}^{N_{c}} \sum_{j=1}^{N_{n}} v_{c n, \mathrm{Y}}\left(r_{i j}\right),
\end{aligned}
$$

includes all electrostatic effective pair potentials. Compared with the primitive model of charged colloids, the coarse-grained model greatly facilitates computational modeling by vastly reducing the number of particles, the microions being included only implicitly through $\kappa$, and by replacing long-range Coulomb pair potentials with shorter-range Yukawa potentials.

Combining the van der Waals pair potential [Eq. (2)] with the electrostatic effective pair potential [Eq. (7)] yields the classic Derjaguin-Landau-Verwey-Overbeek (DLVO) pair potential $[1-5,51,52]$ for charged colloidal mixtures:

$$
v_{\mathrm{DLVO}}(r)=v_{\mathrm{vdW}}(r)+v_{\mathrm{Y}}(r) .
$$

The negative divergence of the van der Waals potential near contact of the particle hard cores renders uncharged or weakly charged colloids and nanoparticles prone to irreversible aggregation. If the particles are sufficiently charged, however, mutual electrostatic repulsion can stabilize a suspension against aggregation.

Figures 2 and 3 illustrate the relative contributions of the van der Waals and Yukawa terms to the colloidcolloid and colloid-nanoparticle effective pair potentials for two different combinations of particle valences. To accentuate the influence of van der Waals interactions, we have here chosen unusually large Hamaker constants, $A_{c c}=A_{c n}=5 \times 10^{-20} \mathrm{~J}$, more typical of metallic particles [4]. In the case of relatively strongly charged colloids and weakly charged nanoparticles (Fig. 2), electrostatic interactions dominate van der Waals interactions, except for very near contact. The total colloid-colloid effective pair potential then has a barrier height far exceeding the typical thermal energy $\left(k_{B} T\right)$. In contrast, for more weakly charged colloids and strongly charged nanoparticles (Fig. 3), electrostatic and van der Waals interactions compete more evenly near contact, resulting in a lower barrier height of $v_{c c}(r)$.

While most of the simulations described in Sec. IV were performed for systems governed by pair potentials typified by Fig. 2, where van der Waals interactions can be neglected, we also simulated a system governed by the pair potentials depicted in Fig. 3. In the latter case, where particles interact via the full DLVO pair potential, it was necessary to cut off the divergence of the van der Waals potential near contact of the particle hard cores. This cutting off was achieved by including shortrange steric interactions, modeled by shifted, repulsive Lennard-Jones pair potentials of the form

$$
v_{\mathrm{LJ}}(r)= \begin{cases}4 \epsilon_{\mathrm{LJ}}\left[\left(\frac{\sigma_{\mathrm{LJ}}}{r}\right)^{12}-\left(\frac{\sigma_{\mathrm{LJ}}}{r}\right)^{6}\right]+\epsilon_{\mathrm{LJ}}, & r<r_{c} \\ 0, & r>r_{c},\end{cases}
$$

where $r_{c}=2^{1 / 6} \sigma_{\mathrm{LJ}}$ and $\sigma_{\mathrm{LJ}}$ and $\epsilon_{\mathrm{LJ}}$ are chosen to yield a total pair potential that is steeply repulsive near core contact and has a well depth of at least $10 k_{B} T$. The required values of $\epsilon_{\mathrm{LJ}}$ are in the range $10^{6}-10^{7} k_{B} T$, closely mimicking a hard-sphere pair potential. In all cases considered here, the colloid-nanoparticle effective pair potential is electrostatically dominated, the barrier height of $v_{c n}(r)$ being $\gg k_{B} T$, precluding adorption of nanoparticles onto colloids. 

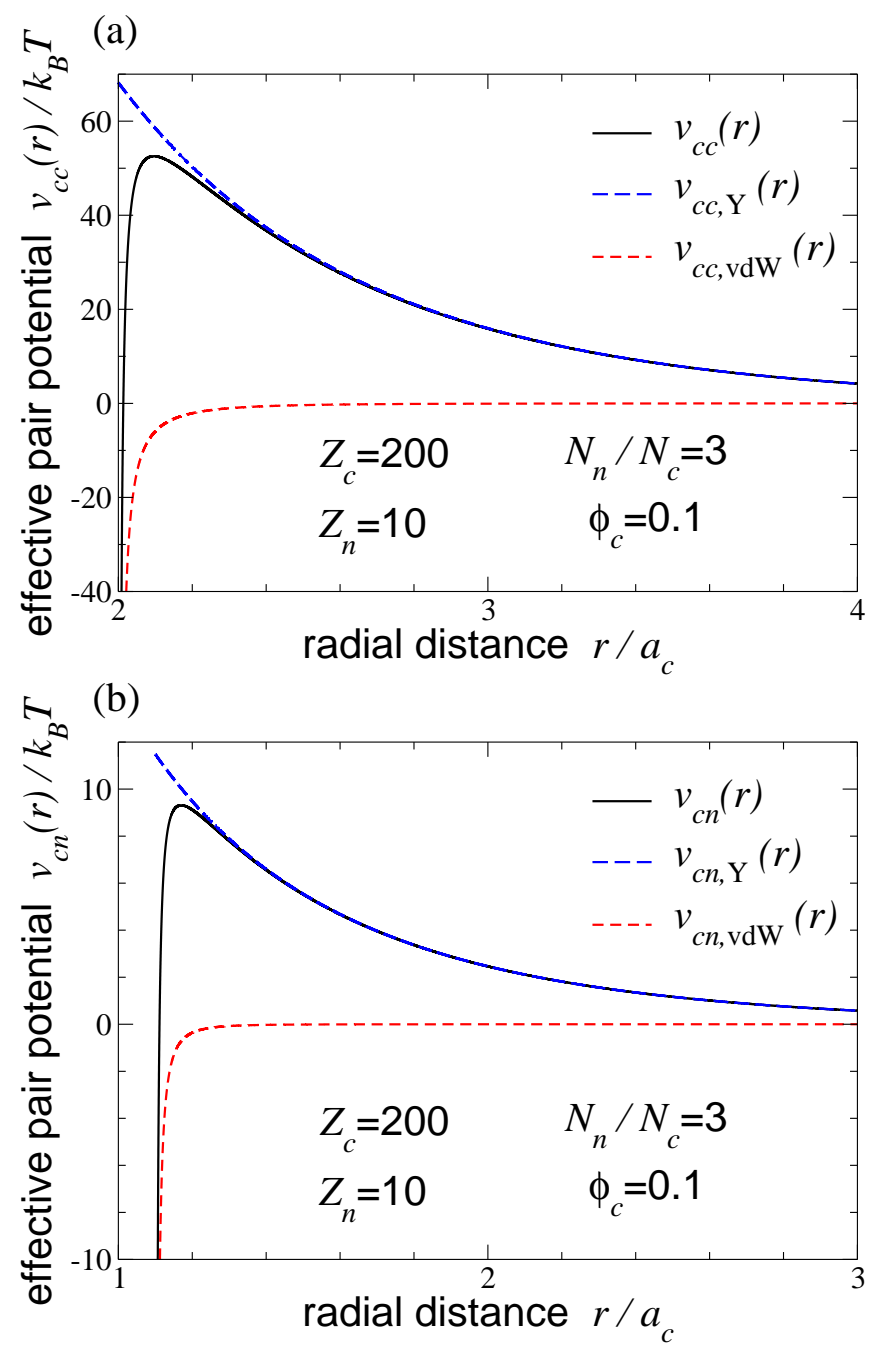

FIG. 2. Effective pair potentials ( $k_{B} T$ units) in deionized (salt-free) mixtures of charged colloids and nanoparticles. (a) Colloid-colloid and (b) colloid-nanoparticle effective pair potentials for colloid radius $a_{c}=50 \mathrm{~nm}$ and valence $Z_{c}=200$; nanoparticle radius $a_{n}=5 \mathrm{~nm}$ and valence $Z_{n}=10$; colloid volume fraction $\phi_{c}=0.1$, number of nanoparticles per colloid $N_{n} / N_{c}=3$, and Hamaker constants $A_{c c}=A_{c n}=5 \times 10^{-20}$ J. The total effective pair potential (solid curves) is the sum of the electrostatic Yukawa potential (dashed curves) and the van der Waals potential (short-dashed curves).

\section{COMPUTATIONAL METHODS}

For the coarse-grained model of charged colloidnanoparticle mixtures described by Eqs. (7)-(11), we performed molecular dynamics (MD) simulations, using LAMMPS $[53,54]$ to integrate (by Verlet's method) Newton's equations of motion. All of our runs were for likecharged (i.e., mutually repulsive) particles. In most of the runs, the particle valences were chosen sufficiently high that electrostatic interactions dominated the effective pair potentials, as exemplified by Fig. 2 . In these cases, since the potential barrier was so high $\left(\gg k_{B} T\right)$,
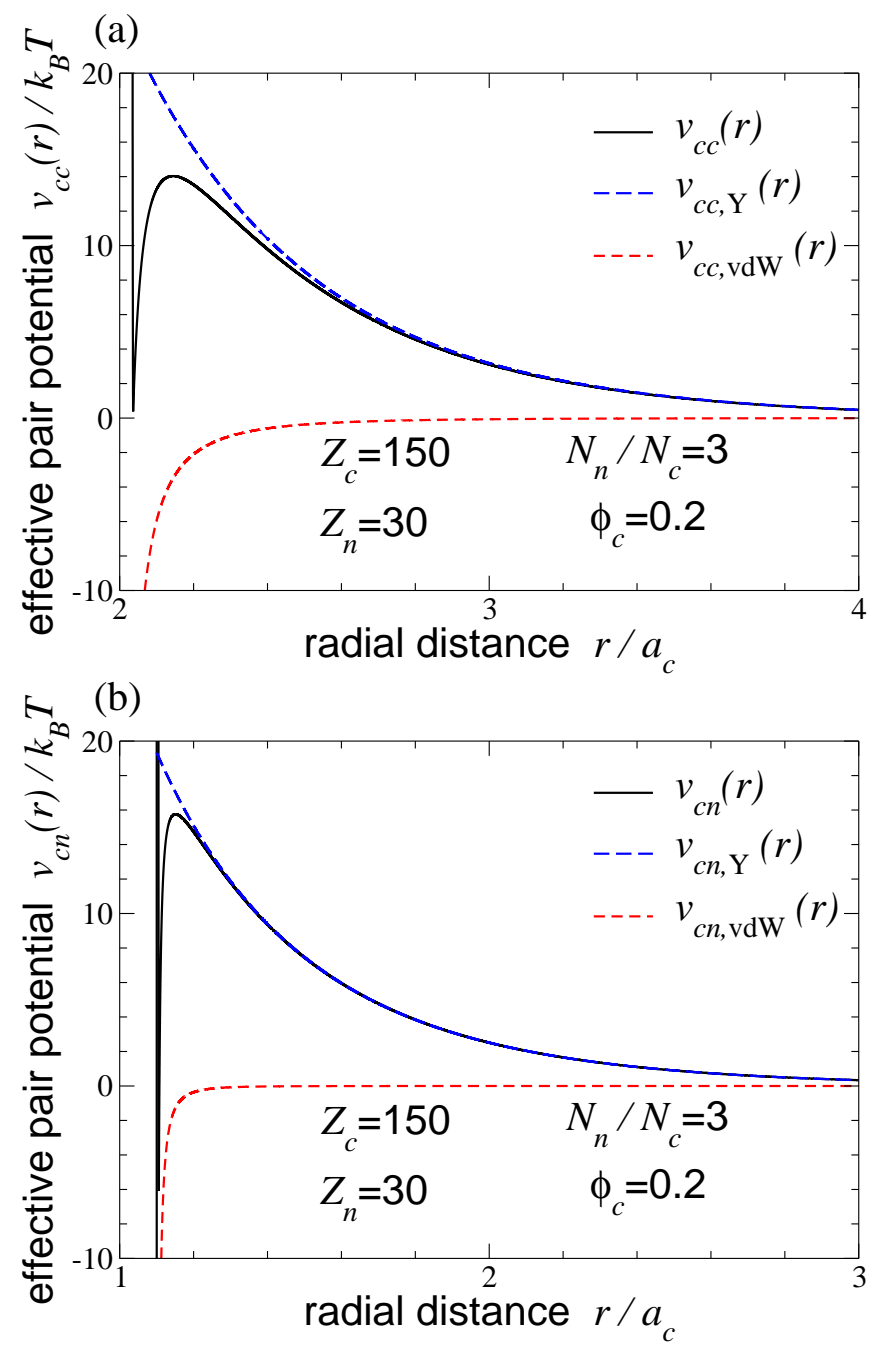

FIG. 3. Same as Fig. 2, but for more weakly charged colloids $\left(Z_{c}=150\right)$, more strongly charged nanoparticles $\left(Z_{n}=30\right)$, and higher colloid volume fraction $\left(\phi_{c}=0.2\right)$. Steric interactions, modeled by short-range, repulsive pair potentials [Eq. (11)], are added to cut off the van der Waals divergence at contact of the particle hard cores. Note the changes of vertical scale.

we were able to neglect van der Waals and steric interactions and use a time step of $10 \mathrm{fs}$. In a few runs, detailed in Sec. IV, we set the colloid valence low enough that van der Waals and steric interactions between the particle hard cores had to be included (see Fig. 3).

The effective pair potentials were truncated at half the box length, ensuring that the cut-off distance exceeded $10 / \kappa$ (i.e., 10 screening lengths) for most runs. The simulations were conducted in the canonical ensemble for fixed numbers of particles in a cubic box of fixed volume, subject to periodic boundary conditions, at an average temperature of $T=293 \mathrm{~K}$, maintained by a Nosé-Hoover thermostat. While this protocol proves efficient for computing equilibrium properties, other methods (e.g., Langevin dynamics) may be more appropriate 


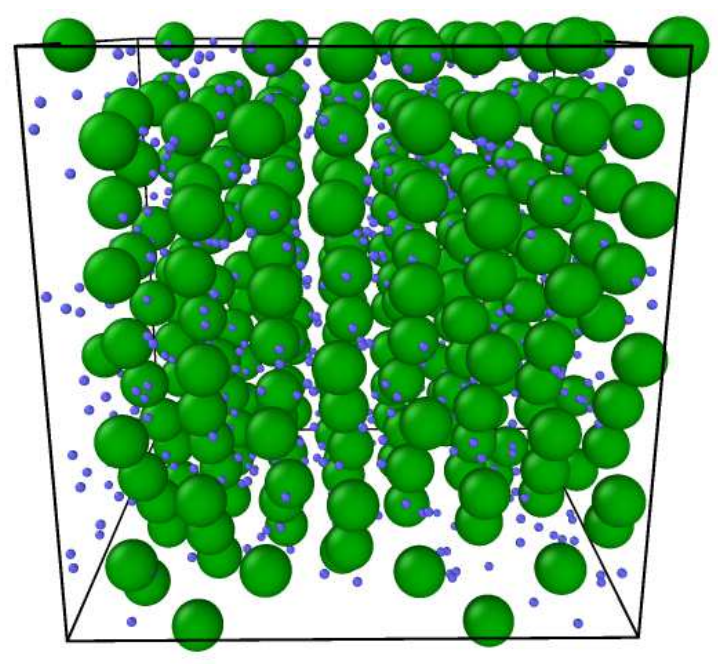

FIG. 4. Snapshot from molecular dynamics simulation of coarse-grained model of a colloid-nanoparticle mixture. Colloids (nanoparticles) are depicted as green (blue) spheres.

for studying dynamical properties. To model aqueous suspensions, we set $\lambda_{B}=0.714 \mathrm{~nm}$, corresponding to $\epsilon \simeq 80$ for water. Variation of the nanoparticle concentration was facilitated by initializing all particles on the sites of a cubic lattice with a basis of up to 16 atoms. After an annealing stage of $10^{5}$ time steps, during which the temperature was steadily ramped down from $1000 \mathrm{~K}$ to $293 \mathrm{~K}$, followed by an equilibration stage of $10^{5}$ steps, we computed structural quantities by averaging over particle trajectories for an additional $10^{6}$ time steps.

From particle configurations, we computed the partial radial distribution functions,

$$
g_{\alpha \beta}(r)=\frac{V}{x_{\alpha} x_{\beta} N^{2}} \sum_{i=1}^{N_{\alpha}} \sum_{j=1}^{N_{\beta}}\left\langle\delta\left(\mathbf{r}+\mathbf{r}_{j}-\mathbf{r}_{i}\right)\right\rangle,
$$

and the partial static structure factors [55],

$$
S_{\alpha \beta}(q)=x_{\alpha} \delta_{\alpha \beta}+\frac{1}{N} \sum_{i=1}^{N_{\alpha}} \sum_{j=1}^{N_{\beta}}\left\langle\frac{\sin \left(q r_{i j}\right)}{q r_{i j}}\right\rangle,
$$

where $q$ is wave vector magnitude, $x_{\alpha}=N_{\alpha} / N$ is the concentration of species $\alpha, \delta(\mathbf{r})$ is the Dirac delta function, $\delta_{\alpha \beta}$ is the Kronecker delta function, the prime on the sum implies exclusion of self-interactions, and angular brackets represent a time average. In computing averages, we sampled independent configurations separated by intervals of $10^{3}$ time steps, thus including a total of $10^{3}$ configurations. As we are particularly interested in the influence of nanoparticles on the colloid-colloid structure factor,

$$
S_{c c}(q)=x_{c}+\frac{1}{N} \sum_{i<j=1}^{N_{c}}\left\langle\frac{\sin \left(q r_{i j}\right)}{q r_{i j}}\right\rangle,
$$

we also define the scaled function,

$$
\frac{S_{c c}(q)}{x_{c}}=1+\frac{1}{N_{c}} \sum_{i<j=1}^{N_{c}}\left\langle\frac{\sin \left(q r_{i j}\right)}{q r_{i j}}\right\rangle,
$$

for direct comparison with the structure factor of the corresponding nanoparticle-free suspension. To test and validate our structural analysis methods, we reproduced previously reported static structure factors of binary Lennard-Jones liquid mixtures [56].

\section{RESULTS AND DISCUSSION}

To explore the influence of charged nanoparticles on structural properties of charge-stabilized colloidal suspensions, we performed a series of simulations over ranges of nanoparticle charge and concentration. Taking care to restrict system parameters to ranges in which the Yukawa effective pair potentials have been validated, we considered only particle valences below established charge renormalization thresholds [57-59], $Z_{c} \lambda_{B} / a_{c} \lesssim 6$ and $Z_{n} \lambda_{B} / a_{n} \lesssim 6$. To reduce the parameter space of these complex systems, we set $N_{c}=500$ and $Z_{c}=200$, fixed the particle radii at $a_{c}=50 \mathrm{~nm}$ and $a_{n}=5 \mathrm{~nm}$, set $z=1$ (monovalent microions) to ensure weak electrostatic coupling $\left(z^{2} \lambda_{B} / a_{c}=0.014, z^{2} \lambda_{B} / a_{n}=0.14\right)$, and considered only salt-free suspensions $\left(n_{s}=0\right)$. We performed runs for colloid volume fractions $\phi_{c}=(4 \pi / 3) n_{c} a_{c}^{3}$ ranging from 0.01 to 0.2 and checked that finite-size effects were negligible by repeating runs for $N_{c}=256$. Figure 4 shows a snapshot from a typical simulation.

Figure 5 presents our simulation data for radial distribution functions in mixtures with nanoparticle valences $Z_{n}=10$ and 30 and a concentration corresponding to 3 nanoparticles per colloid $\left(N_{n}=1500\right)$. Results are shown for colloid volume fractions $\phi_{c}=0.01,0.1$, and 0.2 . The Debye screening constants for these parameters range from $\kappa a_{c}=1$ to $\kappa a_{c}=2$. For reference, results are also shown for $g_{c c}(r)$ from simulations of the one-component model of nanoparticle-free $\left(N_{n}=0\right)$ colloidal suspensions. Given the extreme size asymmetry $\left(a_{n} / a_{c}=0.1\right)$, the total volume fractions of the nanoparticle-containing systems differ only slightly from $\phi_{c}$. Upon addition of nanoparticles, the main peak of $g_{c c}(r)$ drops in height and shifts to shorter separation. With increasing nanoparticle valence, the peak drops further and shifts to yet shorter separations.

Complementary structural trends are observed in wave-vector space. Figure 6 displays the corresponding colloid-colloid static structure factors, computed from particle configurations using Eq. (14), and scaled by a factor of $1 / x_{c}$ [Eq. (15)] for comparison with results for nanoparticle-free suspensions. With increasing valence of added nanoparticles, the main peak of $S_{c c}(q)$ progressively drops in height and shifts to larger $q$. These structural trends indicate that doping a charge-stabilized 
(a)

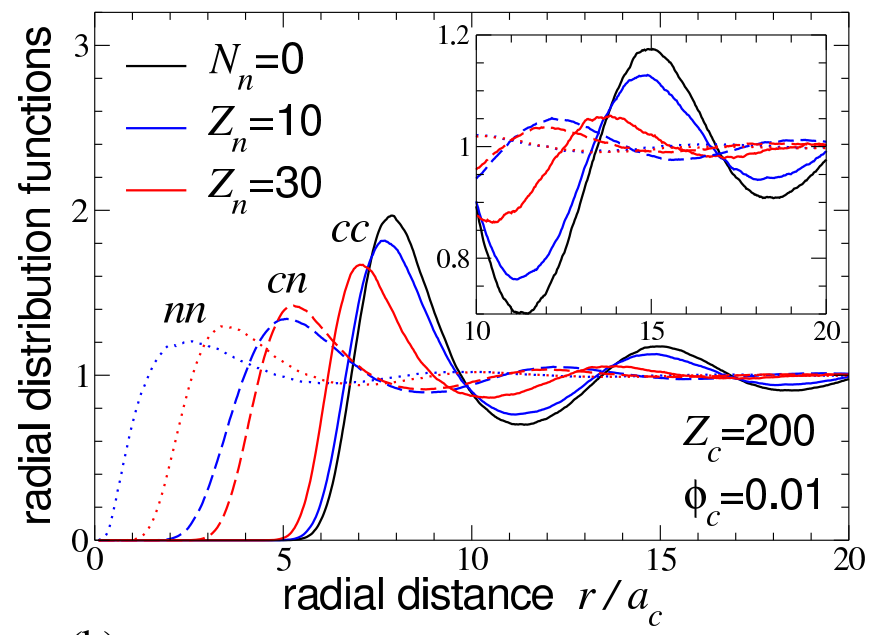

(b)

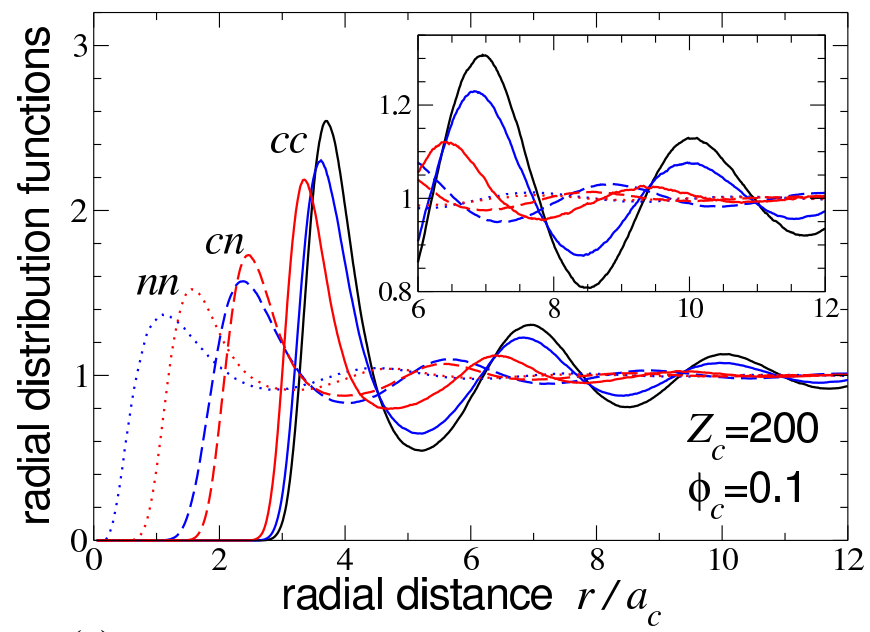

(c)

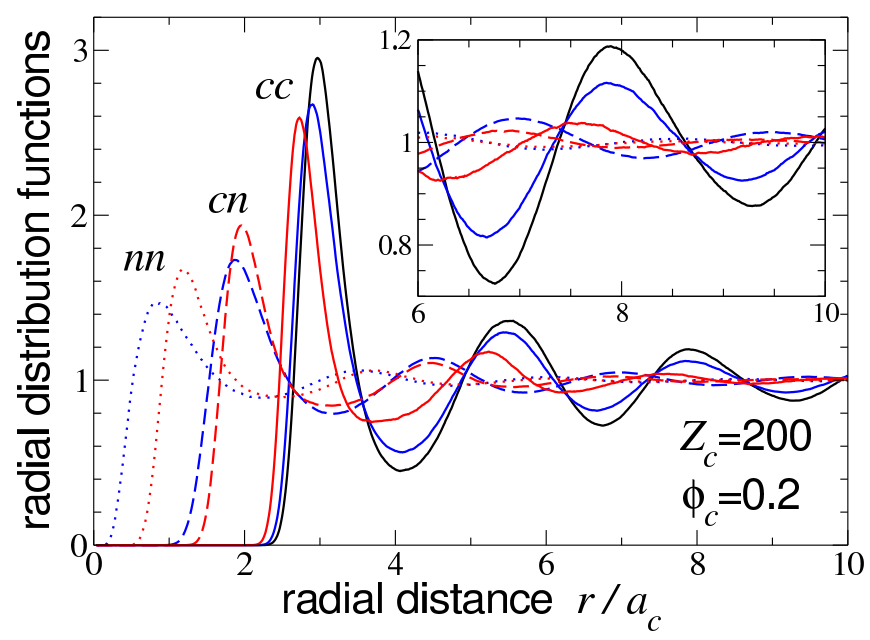

FIG. 5. Radial distribution functions from MD simulations of model salt-free mixtures of like-charged colloids and nanoparticles, interacting via effective pair potentials typical of those in Fig. 2. Results are shown for colloid number $N_{c}=500$, radius $a_{c}=50 \mathrm{~nm}$, and valence $Z_{c}=200$; nanoparticle number $N_{n}=1500$, radius $a_{n}=5 \mathrm{~nm}$, and valences $Z_{n}=10$ (blue curves) and 30 (red curves); and colloid volume fractions $\phi_{c}=0.01$ (a), 0.1 (b), and 0.2 (c). For comparison, $g_{c c}(r)$ of nanoparticle-free $\left(N_{n}=0\right)$ suspensions are also shown (black curves). Solid, dashed, and dotted curves, whose main peaks are labeled $c c, c n$, and $n n$, represent $g_{c c}(r), g_{c n}(r)$, and $g_{n n}(r)$, respectively. Insets magnify long-ranged behavior. (a)

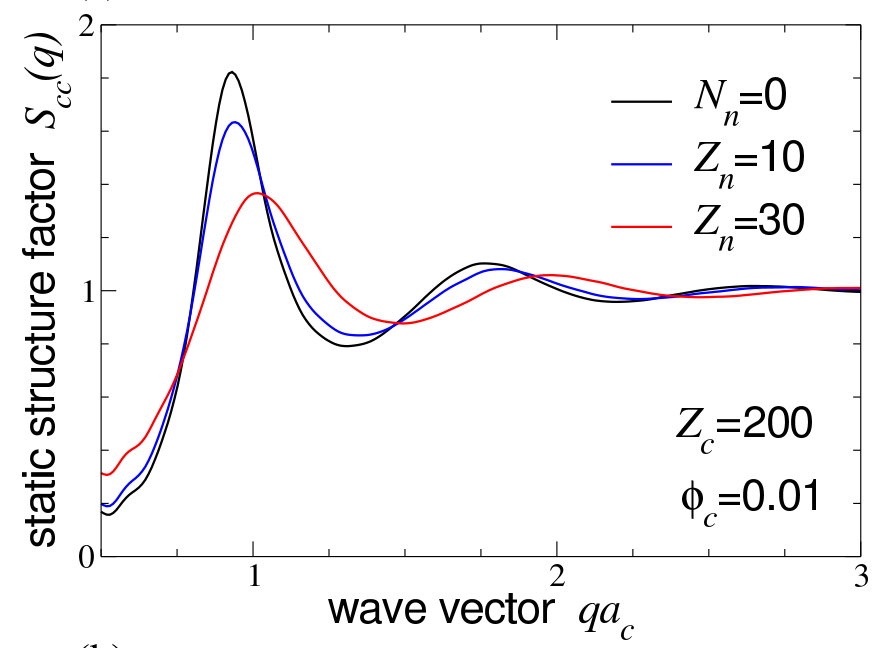

(b)

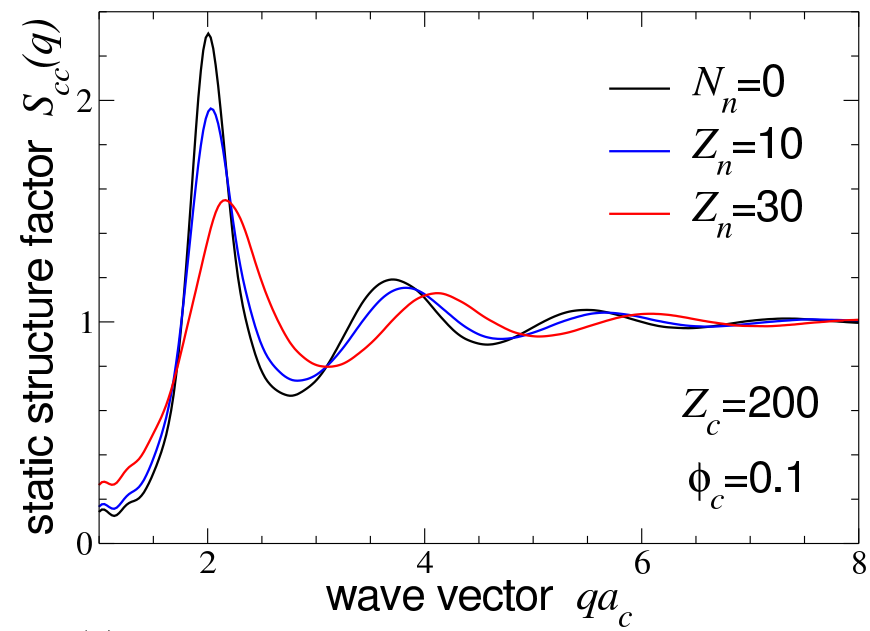

(c)

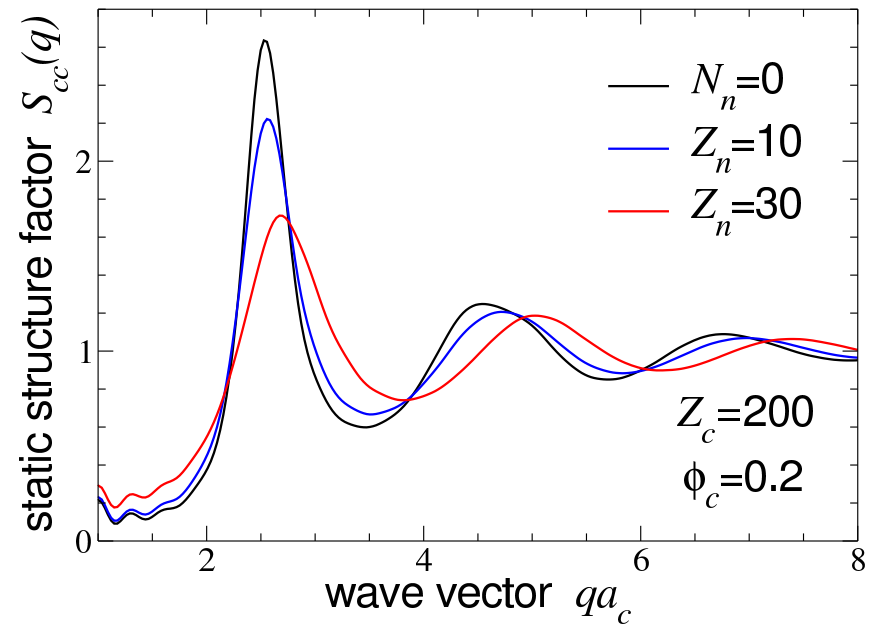

FIG. 6. Colloid-colloid static structure factors, scaled by a factor of $1 / x_{c}$ [Eq. (15)], corresponding to radial distribution functions in Fig. 5 with colloid volume fractions $\phi_{c}=0.01$ (a), 0.1 (b), and $0.2(\mathrm{c})$. 
(a)

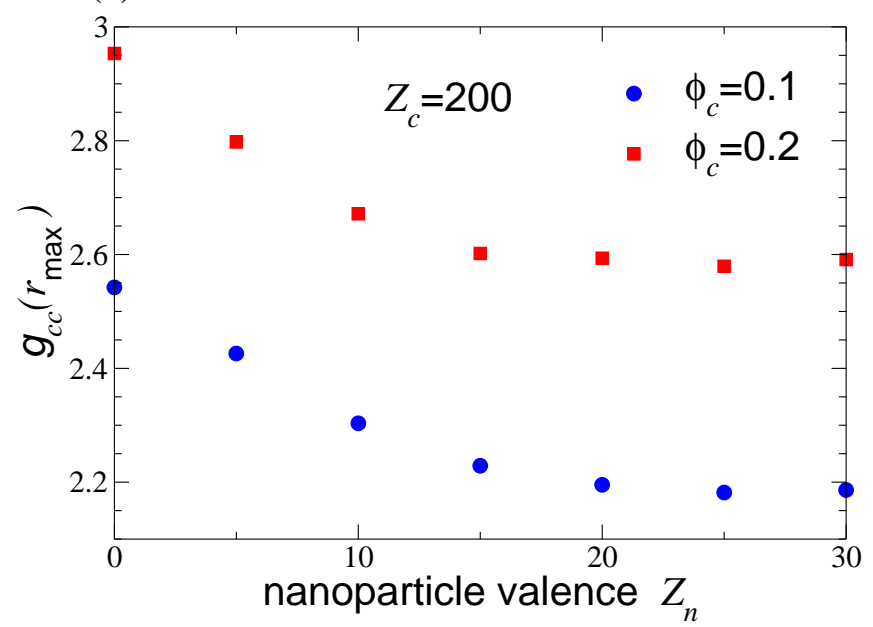

(b)

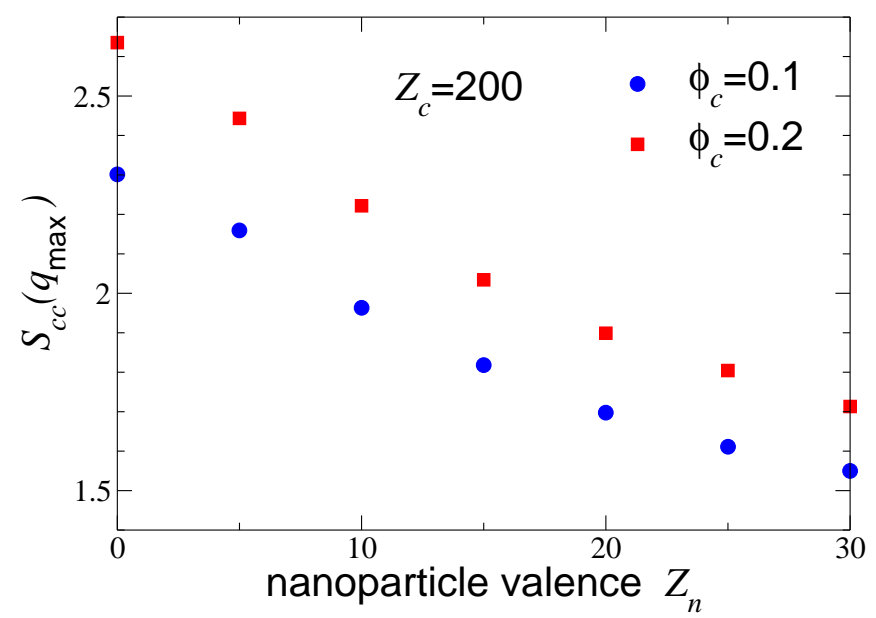

(c)

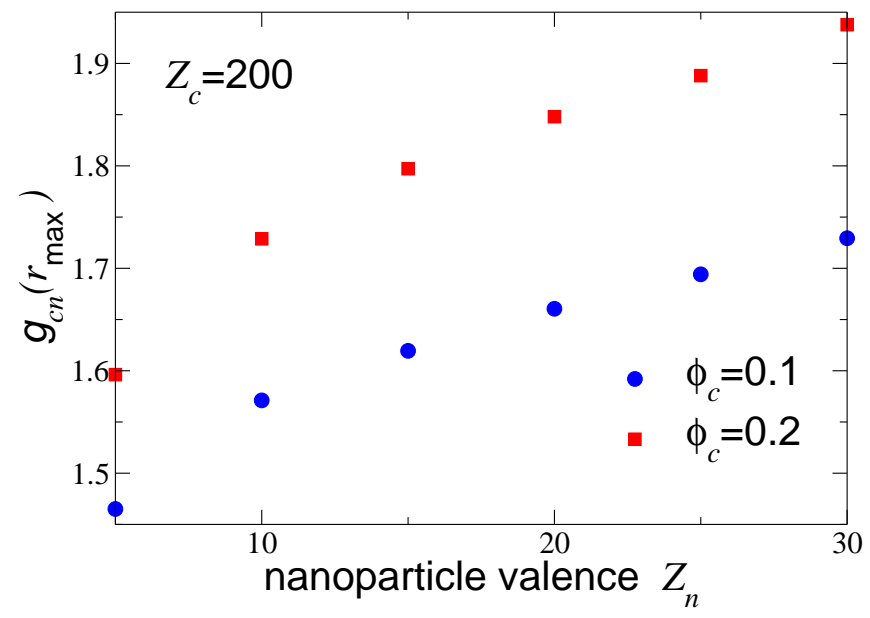

FIG. 7. Height of main peak of (a) colloid-colloid radial distribution function (Fig. 5), (b) colloid-colloid static structure factor (Fig. 6), and (c) colloid-nanoparticle radial distribution function (Fig. 5) vs. nanoparticle valence $Z_{n}$ for colloid number $N_{c}=500$, radius $a_{c}=50 \mathrm{~nm}$, and valence $Z_{c}=200$; nanoparticle number $N_{n}=1500$ and radius $a_{n}=5 \mathrm{~nm}$; and colloid volume fractions $\phi_{c}=0.1$ and 0.2 . (a)

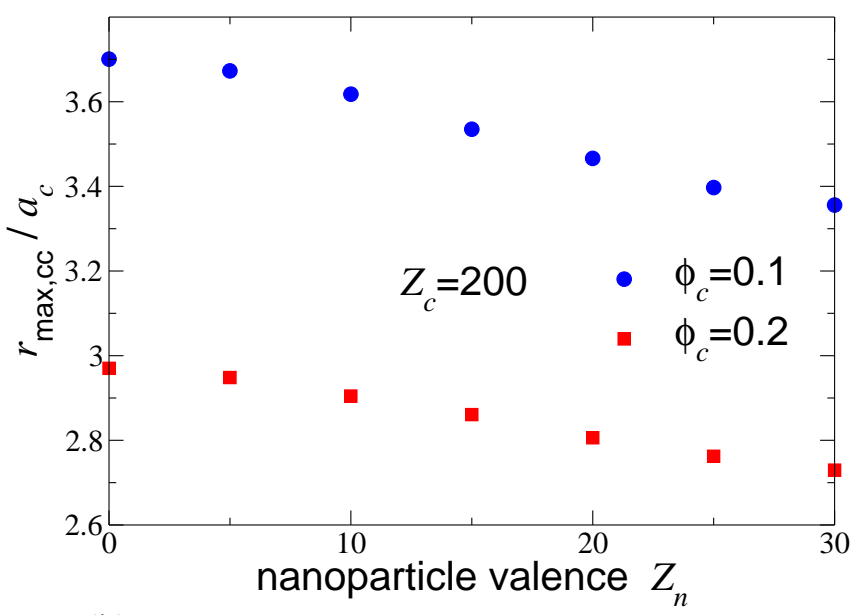

(b)

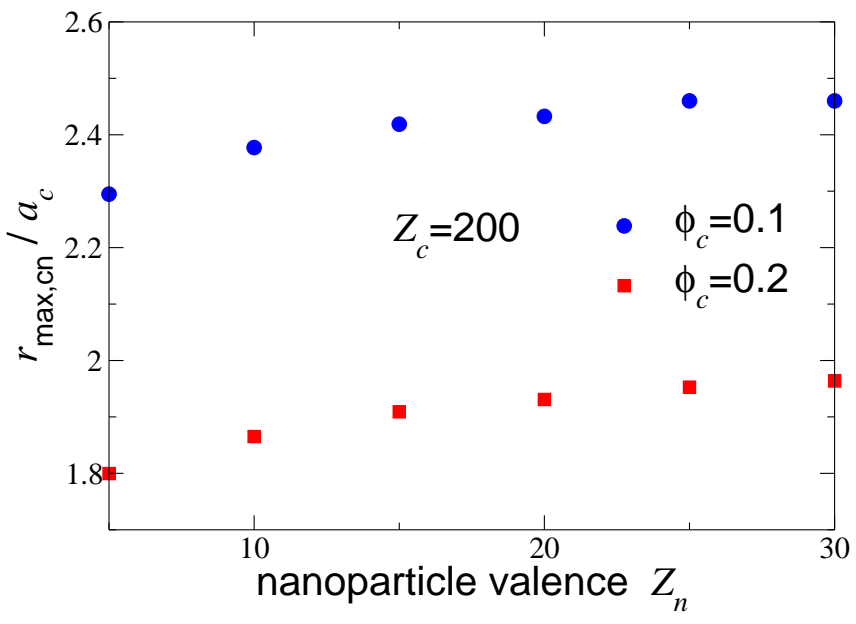

FIG. 8. Position of main peak of (a) colloid-colloid and (b) colloid-nanoparticle radial distribution functions (Fig. 5) vs. nanoparticle valence $Z_{n}$ for colloid number $N_{c}=500$, radius $a_{c}=50 \mathrm{~nm}$, and valence $Z_{c}=200$; nanoparticle number $N_{n}=1500$ and radius $a_{n}=5 \mathrm{~nm}$; and colloid volume fractions $\phi_{c}=0.1$ and 0.2 .

colloidal suspension with charged nanoparticles tends to weaken colloid-colloid correlations.

Also shown in Fig. 5 are results for the colloidnanoparticle and nanoparticle-nanoparticle radial distribution functions, revealing the nanoparticle correlations. With increasing nanoparticle valence, the main peaks of both $g_{c n}(r)$ and $g_{n n}(r)$ rise in height and shift to longer separations. These variations are consistent with the stronger electrostatic repulsion between colloids and nanoparticles and between nanoparticle pairs with increasing $Z_{n}$. Since $g_{c n}(r)$ is proportional to the probability of finding a nanoparticle a radial distance $r$ from the center a given colloid, a sharper main peak reveals a slight tendency for nanoparticles to accumulate near the colloids. Therefore, the colloids facilitate ordering of nanoparticles as well as enhance correlations between nanoparticles. Weakening of colloid-colloid correlations 
(a)

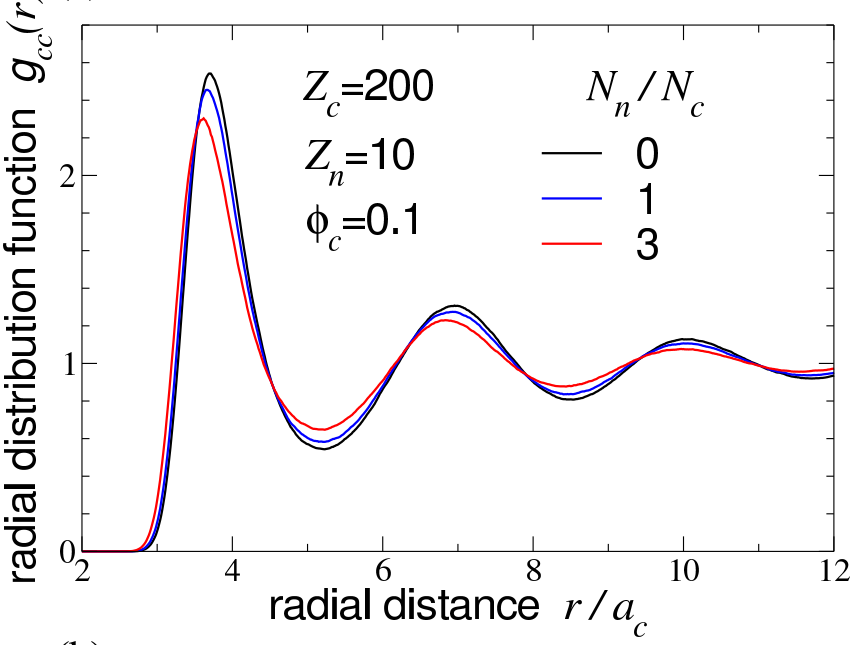

(b)

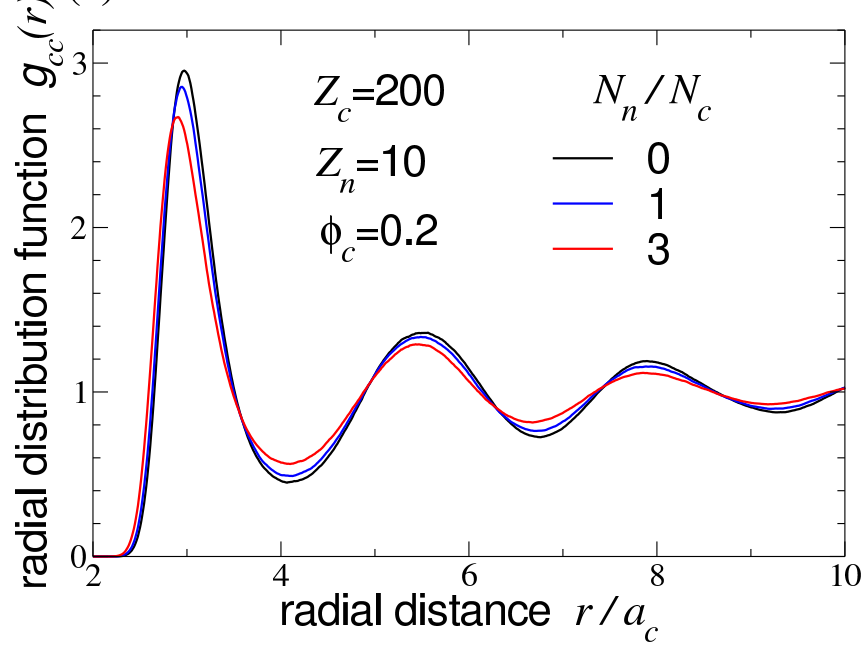

FIG. 9. Colloid-colloid radial distribution functions in saltfree mixtures of charged colloids and nanoparticles with colloid volume fractions $\phi_{c}=0.1$ (a) and 0.2 (b). The colloid and nanoparticle valences are $Z_{c}=200$ and $Z_{n}=10$ and the number of nanoparticles per colloid, $N_{n} / N_{c}$, varies from 0 (black) to 1 (blue) to 3 (red). Other system parameters are the same as in Fig. 5. With increasing nanoparticle concentration, colloid-colloid correlations become weaker, as reflected by the decreasing height of the main peak of $g_{c c}(r)$.

upon increasing nanoparticle charge is thus accompanied by a modest strengthening of both colloid-nanoparticle and nanoparticle-nanoparticle correlations. It is important to emphasize that this gathering of nanoparticles around colloids, which is driven by electrostatic repulsion, differs fundamentally from the nanoparticle adsorption and haloing observed in experiments [6-10], which is associated with van der Waals attraction.

The structural trends exhibited in Figs. 5 and 6 are systematically organized in Figs. 7 and 8, which plot the main peak heights and positions of the radial distribution functions and static structure factors over a range of nanoparticle valence for two colloid volume fractions. (a)

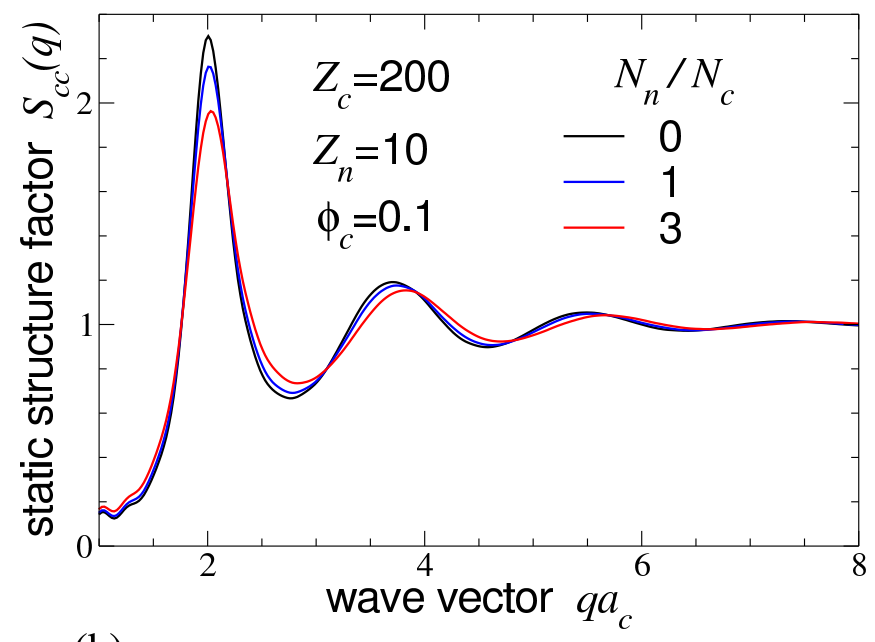

(b)

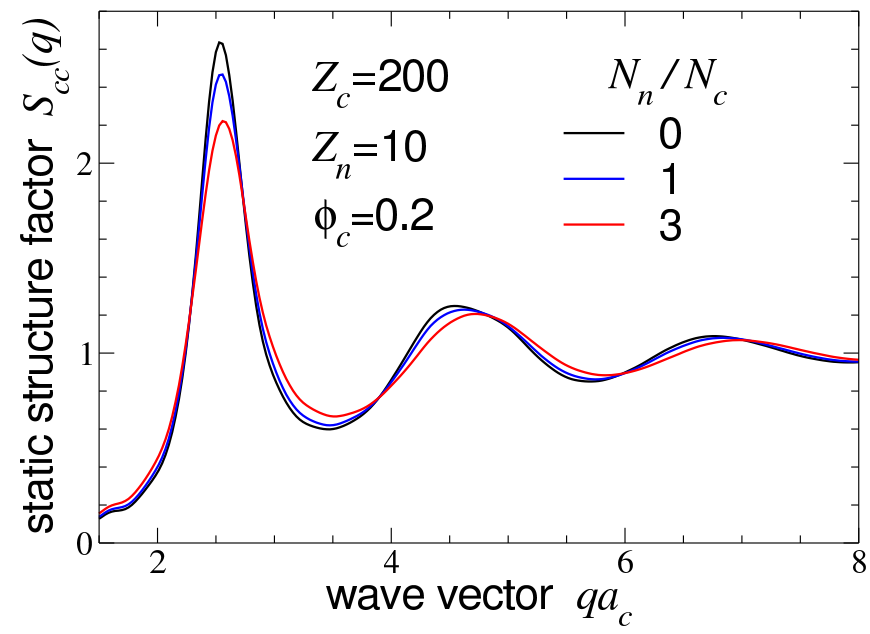

FIG. 10. Scaled colloid-colloid static structure factors corresponding to radial distribution functions in Fig. 9 with colloid volume fractions $\phi_{c}=0.1(\mathrm{a})$ and $0.2(\mathrm{~b})$.

With increasing $Z_{n}$, the main peaks of $g_{c c}(r)$ and $S_{c c}(q)$ progressively drop in height, while the peak positions $r_{\max }$ for $g_{c c}(r)$ and $q_{\max }$ for $S_{c c}(q)$ - shift to smaller $r$ and larger $q$, respectively. At the same time, the main peaks of $g_{c n}(r)$ and $g_{n n}(r)$ monotonically increase in height and shift to larger $r$. Secondary peaks show the same trends, consistent with weakening of colloid-colloid correlations and strengthening of colloid-nanoparticle correlations with increasing nanoparticle valence.

Figures 9 and 10 further illustrate the dependence of $g_{c c}(r)$ and $S_{c c}(q)$, respectively, on the concentration of nanoparticles, which varies from 0 to 3 nanoparticles per colloid. The structural trends observed upon increasing $N_{n}$ at fixed $Z_{n}$ are qualitatively similar to those resulting from increasing $Z_{n}$ at fixed $N_{n}$. Collectively, these results demonstrate that adding charged nanoparticles tends to weaken correlations between charged colloids.

The physical mechanism by which charged nanoparticles weaken correlations between colloids of like charge 

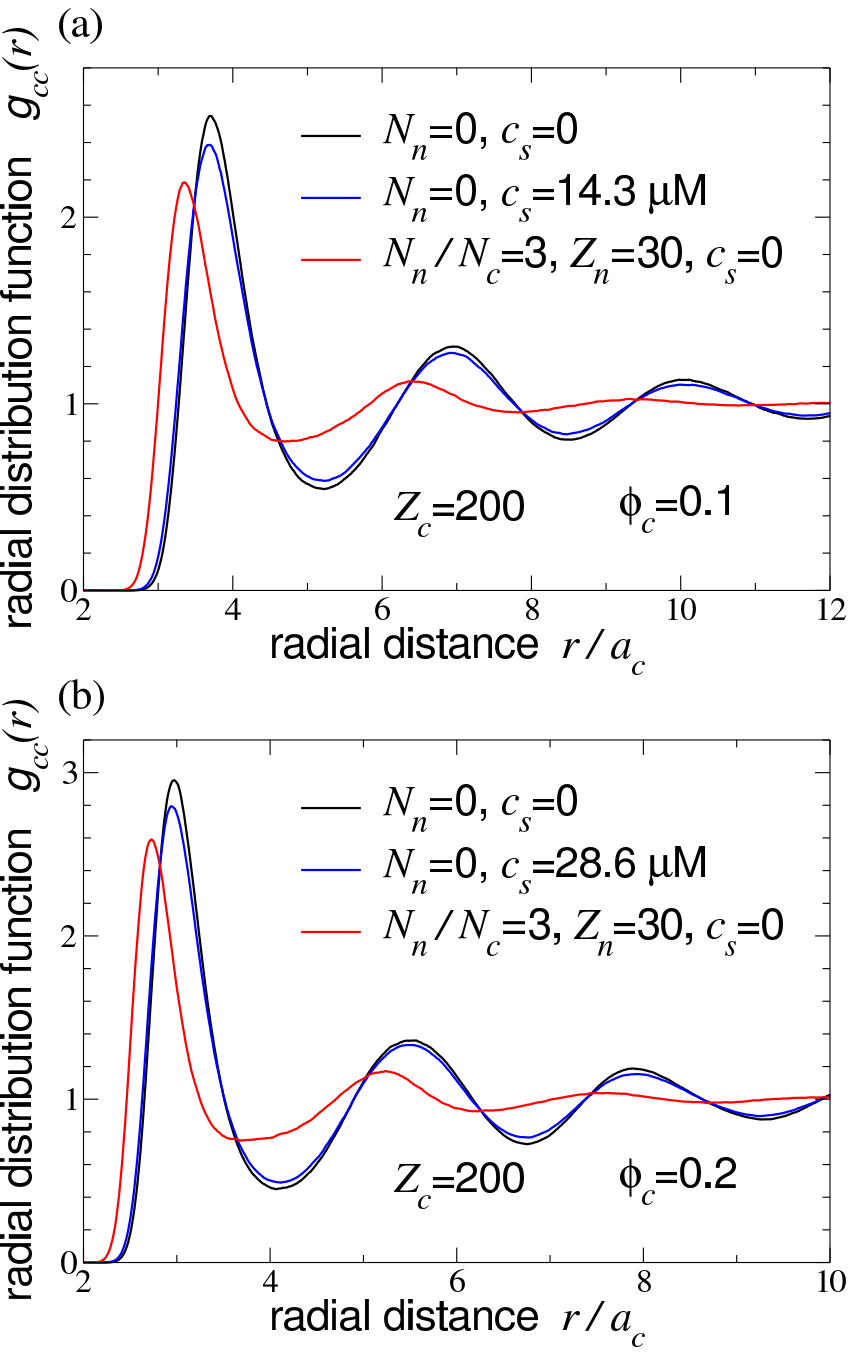

FIG. 11. Colloid-colloid radial distribution function in a colloid-nanoparticle mixture (red curves), with colloid and nanoparticle valences $Z_{c}=200$ and $Z_{n}=30$, and in a nanoparticle-free suspension (blue curves) to which salt is added at concentration sufficient to yield the same Debye screening constant. For reference, $g_{c c}(r)$ is shown also for the corresponding salt- and nanoparticle-free suspensions (black curves). For colloid volume fractions $\phi_{c}=0.1$ (a) and 0.2 (b), the required salt concentrations are $c_{s}=14.3 \mu \mathrm{M}$ and $28.6 \mu \mathrm{M}$. Other system parameters are the same as in Fig. 5.

is subject to interpretation. One possible perspective is that a large charge asymmetry energetically favors configurations in which nanoparticles accumulate around the colloids, since the condition $\left|Z_{n}\right| \ll\left|Z_{c}\right|$ (or $\left.\left|Z_{c} Z_{n}\right| \ll Z_{c}^{2}\right)$ advantages colloid-nanoparticle interactions over colloid-colloid interactions. The fact that the peak of $g_{c n}(r)$ lies closer to the colloid center for smaller $Z_{n}$ (greater charge asymmetry) and is higher and sharper for larger $Z_{n}$, when electrostatic coupling between colloids and nanoparticles is stronger, may support this perspective. However, the relative contributions of energy and entropy to the total free energy are difficult to assess (a)

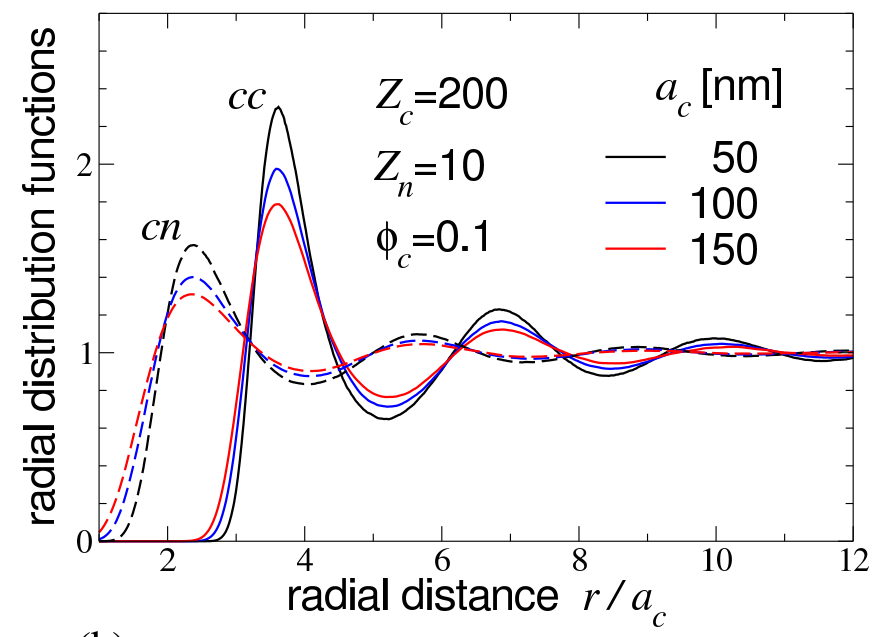

(b)

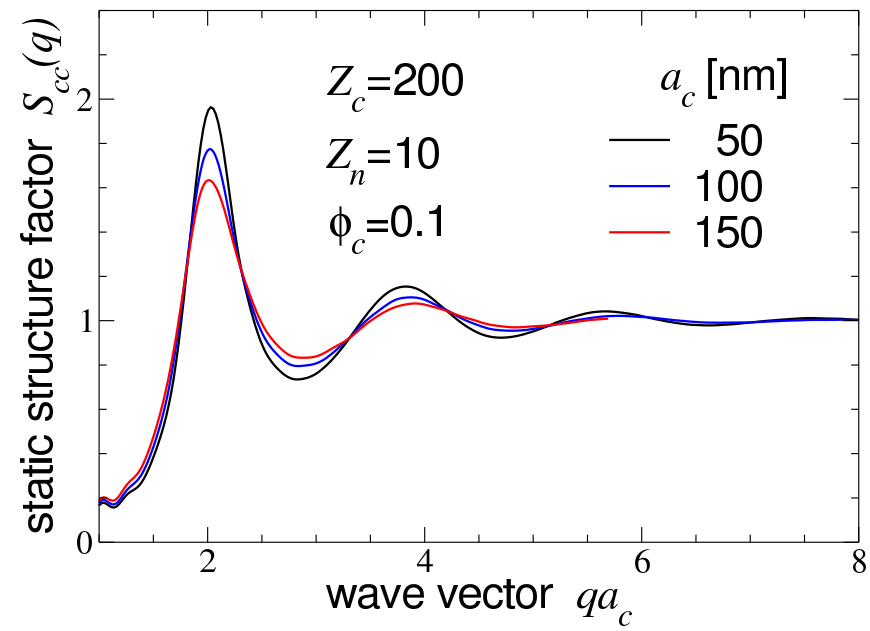

FIG. 12. Variation of colloid-colloid and colloid-nanoparticle radial distribution functions (a) and scaled colloid-colloid static structure factor (b) with colloid radius $\left(a_{c}=\right.$ $50,100,150 \mathrm{~nm})$ for fixed nanoparticle radius $\left(a_{n}=5 \mathrm{~nm}\right)$, valences $\left(Z_{c}=200, Z_{n}=10\right)$, and colloid volume fraction $\left(\phi_{c}=0.1\right)$. In panel (a), solid and dashed curves, whose main peaks are labeled $c c$ and $c n$, represent $g_{c c}(r)$ and $g_{c n}(r)$, respectively.

and may depend on specific system parameters.

Another conceivable interpretation is that charged nanoparticles may act to enhance the screening of electrostatic interactions between colloids. To test this possibility, we performed additional simulations for colloidal suspensions that are free of nanoparticles, but contain salt of sufficient concentration to yield the same Debye screening constant as in a salt-free suspension with charged nanoparticles. Figure 11 compares the colloid-colloid radial distribution functions for these two systems. Correlations between colloids in the nanoparticle-containing, salt-free suspension are seen to be considerably weaker than in the salt-containing, nanoparticle-free suspension of the same screening length. The peaks of $g_{c c}(r)$ 
(a)

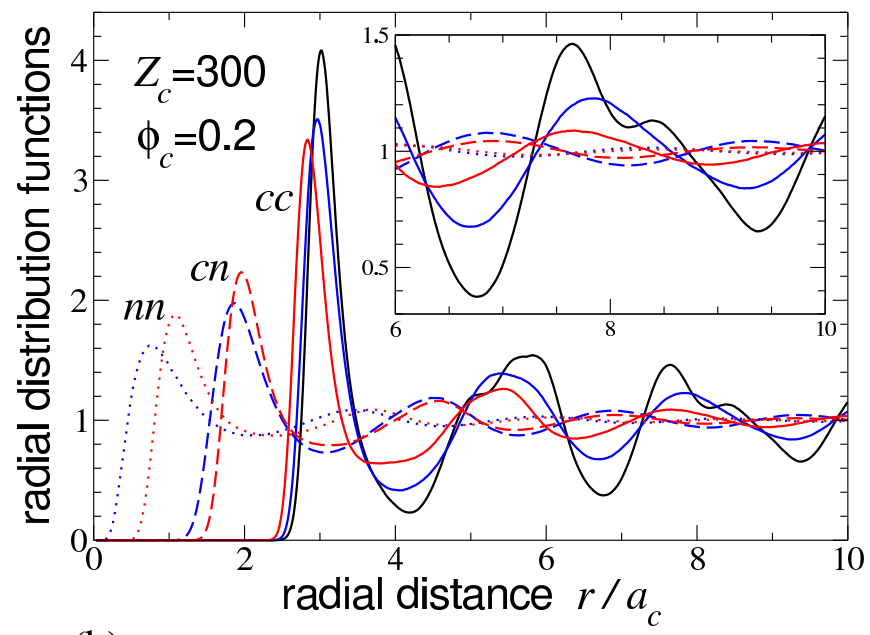

(b)

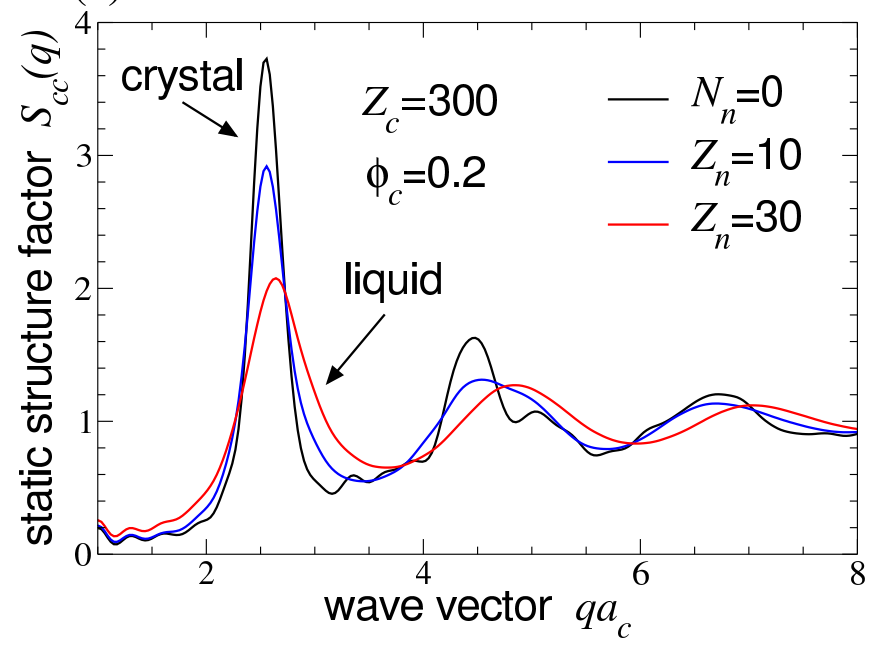

FIG. 13. (a) Radial distribution functions and (b) scaled colloid-colloid static structure factors for suspensions of $N_{c}=$ 500 colloids of valence $Z_{c}=300$ and volume fraction $\phi_{c}=0.2$, both without added nanoparticles (black) and with $N_{n}=$ 1500 nanoparticles of valence $Z_{n}=10$ (blue) or 30 (red). In panel (a), solid, dashed, and dotted curves, whose main peaks are labelled $c c, c n$, and $n n$, represent $g_{c c}(r), g_{c n}(r)$, and $g_{n n}(r)$, respectively. Inset magnifies long-ranged behavior. Adding 3 nanoparticles per colloid of valence $Z_{n}=10$ weakens correlations (blue curves). Increasing $Z_{n}$ to 30 further weakens correlations (red curves), inducing melting of the colloidal crystal, as reflected by the drop in height of the main peak of $S_{c c}(q)$.

are lower and at shorter distances in the nanoparticlecontaining suspension than in the salt-containing suspension. This comparison demonstrates that ions confined to the surfaces of charged nanoparticles have a significantly stronger screening effect, and thus overall damping effect on correlations, than an equal number of free salt ions. Furthermore, this interpretation is consistent with conclusions drawn from an effective interaction theory, recently developed by one of us [60]. This theory, which is based on linear-response and random-phase approximations, maps a charged colloid-nanoparticle mixture onto a coarse-grained, one-component model of pseudo-colloids governed by an effective electrostatic pair potential

$$
v_{\text {eff }}(r) \propto \frac{e^{-\kappa_{\text {eff }} r}}{r},
$$

involving a nanoparticle-enhanced, effective screening constant

$$
\kappa_{\mathrm{eff}}=\sqrt{\kappa^{2}+4 \pi \lambda_{B} \frac{Z_{n, \mathrm{eff}}^{2} n_{n}}{1-\phi^{\prime}}}
$$

[compare with $\kappa$, defined in Eq. (8)], where

$$
Z_{n, \mathrm{eff}}=\frac{e^{\kappa a_{n}}}{1+\kappa a_{n}} Z_{n}
$$

represents an effective valence of the nanoparticles, and $\phi^{\prime}=(4 \pi / 3) n_{c}\left(a_{c}+a_{n}\right)^{3}$ is the volume fraction from which the nanoparticles are excluded by the colloids. As confirmed by our simulations and the simulations of ref. [60], charged nanoparticles increase the effective screening constant much more efficiently than microions. For example, a nanoparticle of valence $Z_{n}=10$ contributes more to screening than 10 free microions. We note that this interpretation differs somewhat from one recently suggested by Ojeda-Mendoza et al. [36], who attributed the influence of smaller particles in binary mixtures of charged colloids to an electrostatically-enhanced depletion attraction, albeit for size and charge asymmetries less extreme than considered here.

In addition to varying the charge asymmetry between colloids and nanoparticles, we also performed additional simulations to explore the influence of size asymmetry on structure. Figure 12 shows radial distribution functions and static structure factors for three different colloid radii, $a_{c}=50,100$, and $150 \mathrm{~nm}$, at fixed colloid volume fraction, $\phi_{c}=0.1$. With increasing colloid size, colloid-colloid and colloid-nanoparticle correlations become weaker, as signaled by the drop in height of the main peaks of $g_{c c}(r), g_{c n}(r)$, and $S_{c c}(q)$. This trend can be at least partially explained by the decrease in electrostatic coupling strength, $\lambda_{B} / a_{c}$, and the decrease in screening constant $\kappa$ [Eq. (8)] that results from increasing $a_{c}$ at fixed $\phi_{c}$. This behavior is opposite, however, what would be expected of uncharged colloids, around which charged nanoparticles tend to accumulate to lower their potential energy, the accumulation growing stronger the larger the volume displaced by the colloids.

We note in passing that, since the like-charged particles considered here interact via repulsive Yukawa forces that prevent their steeply repulsive cores from coming close to contact, conventional excluded-volume arguments regarding depletion of nanoparticles from the spaces between colloids do not cleanly apply here. For this reason, the extent to which weakening of colloidal correlations can be directly associated with depletion-induced attraction is unclear and left open by our study. In the limit of 
uncharged particles, however, the system clearly reduces to an asymmetric mixture of hard spheres, for which density-functional theories of effective depletion-induced interactions have been developed [61-64]. The crossover between a parameter regime dominated by electrostatic interactions and a regime dominated by excluded-volume interactions in charged hard-sphere mixtures is not well understood and deserves future study.

A potential application of nanoparticle doping is illustrated in Fig. 13, which shows structural data for systems with a higher colloidal valence of $Z_{c}=300$. Suspensions of macroions of sufficient charge can self-assemble into ordered crystals. According to the Hansen-Verlet freezing criterion [65], the solid phase is stable when the height of the main peak of $S_{c c}(q)$ exceeds about 2.85. Correspondingly, $g_{c c}(r)$ exhibits pronounced peaks at lattice coordination distances. When added to a colloidal crystal, nanoparticles may weaken colloidal interparticle correlations to the point of inducing melting of the crystal. From inspection of Fig. 13, the nanoparticle-free suspension is clearly in a stable solid phase, with $S_{c c}\left(q_{\max }\right) \simeq 3.8$. The positions of the peaks of $g_{c c}(r)$ and $S_{c c}(q)$ are consistent with fcc crystal symmetry. Upon adding nanoparticles in a 3:1 ratio $\left(N_{n} / N_{c}=3\right)$, the colloidal correlations are substantially weakened. For nanoparticles of valence $Z_{n}=10$, the main peak height of the structure factor drops to $S_{c c}\left(q_{\max }\right) \simeq 2.9$, placing the solid on the verge of melting. The mixture of nondiffusing colloids and mobile nanoparticles is reminiscent of a superionic lattice (or sublattice melt) observed in atomic alloys. At higher nanoparticle valence $\left(Z_{n}=30\right)$, colloidal correlations are furthered weakened and the structure factor clearly reveals that the crystal has melted.

Finally, to elucidate the interplay between electrostatic and van der Waals interactions in mixtures of likecharged colloids and nanoparticles, we performed additional simulations for systems interacting via the pair potentials shown in Fig. 3. Reducing the colloid valence from $Z_{c}=200$ to 150 significantly lowers the barrier height of the total (DLVO) effective pair potential $v_{c c}(r)$, rendering bulk stability much more sensitive to the balance between competing repulsive electrostatic and attractive van der Waals interactions. Figure 14 shows representative results for radial distribution functions obtained from runs of the same duration, but for $10^{7}$ steps, with the step now shortened to $1 \mathrm{fs}$, as necessitated by the rapid spatial variations of the pair potentials.

In a pure, nanoparticle-free suspension, represented by the dashed curve in Fig. 14, the barrier of $v_{c c}(r)$ is sufficiently high $\left(\sim 20 k_{B} T\right)$ that the suspension is largely stable (or metastable) against van der Waals attractioninduced aggregation. Although a small fraction of colloid pairs have aggregated, as indicated by a narrow peak in $g_{c c}(r)$ near colloid-colloid contact $\left(r=2 a_{c}\right)$, most particles remain dispersed. Adding nanoparticles significantly lowers the barrier height to $\sim 14 k_{B} T$ and precipitates aggregation of colloids, as signaled by a sharp peak in $g_{c c}(r)$ at contact (solid $c c$ curve in Fig. 14). Secondary

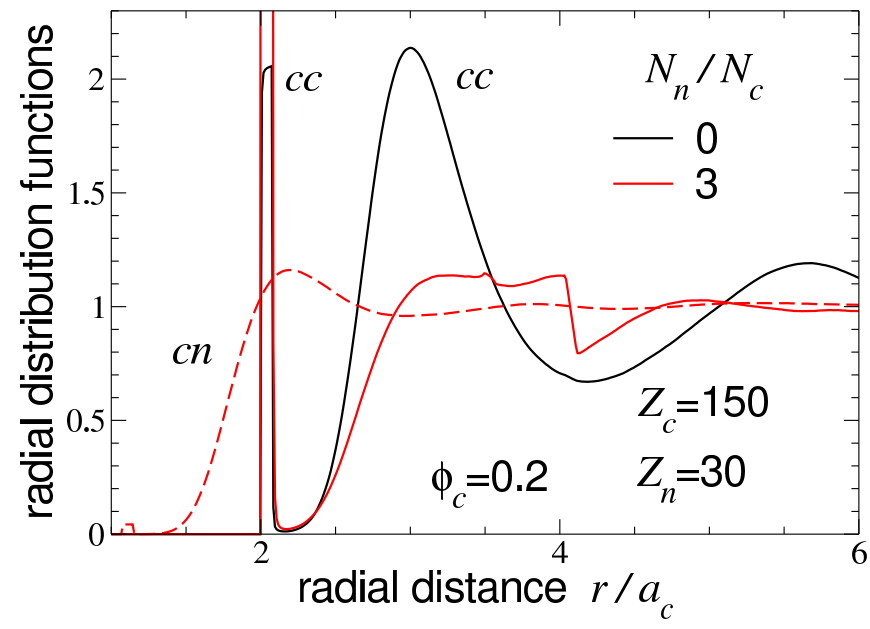

FIG. 14. Colloid-colloid (cc) and colloid-nanoparticle (cn) radial distribution functions (solid and dashed red curves, respectively) from our MD simulations of a salt-free mixture of like-charged colloids (valence $Z_{c}=150$, volume fraction $\phi_{c}=0.2$ ) and nanoparticles (valence $Z_{n}=30$ ), with three nanoparticles per colloid, interacting via effective pair potential of Fig. 3. Also shown, for reference, is $g_{c c}(r)$ for the corresponding nanoparticle-free $\left(N_{n}=0\right)$ suspension (solid black curve). In the presence of van der Waals interactions, charged nanoparticles facilitate aggregation of weakly charged colloids, as reflected in the sharp peak of $g_{c c}(r)$ near hard-core contact $\left(r=2 a_{c}\right)$ and smaller secondary peaks.

peaks at larger separations reflect the onset of clustering. Most prominent is the peak at $r=4 a_{c}$, corresponding to three aggregated colloids in a linear configuration. At the same time, electrostatic repulsion between colloids and nanoparticles gives a barrier height in $v_{c n}(r)$ of $\sim 16 k_{B} T$ - sufficiently strong to prevent adsorption of nanoparticles onto the colloid surfaces, as seen from the absence of a peak in $g_{c n}(r)$ at colloidnanoparticle contact $\left(r=a_{c}+a_{n}\right)$. The nanoparticles, being free to screen electrostatic interactions, thus act to weaken colloid-colloid correlations and facilitate aggregation. Figure 15 shows snapshots from these simulations in which an aggregated pair of colloids is indicated by drawing a bond joining the two particles. The figure illustrates the higher concentration of aggregated pairs in the mixture, as well as the dispersed distribution of nanoparticles. It should be noted that, since colloidal aggregation is essentially irreversible, the systems modeled in this case are not necessarily in thermodynamic equilibrium. Nevertheless, our results clearly show the destabilizing influence of charged nanoparticles.

\section{CONCLUSIONS}

In summary, by performing a computer simulation study of a coarse-grained model of charged colloidnanoparticle mixtures, in which particles interact via physically consistent effective pair potentials, we have ex- 
(a)



(b)

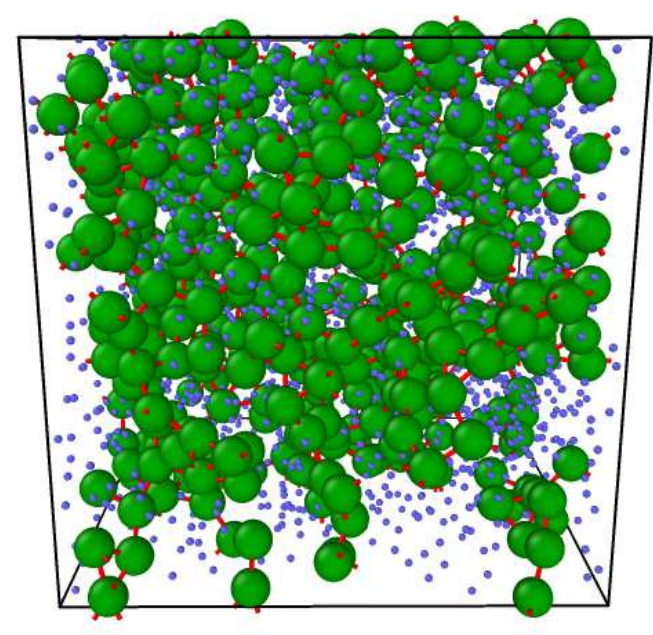

FIG. 15. Snapshots from simulations of (a) colloidnanoparticle mixture and (b) pure (nanoparticle-free) colloidal suspension with particles of valences $Z_{c}=150$ and $Z_{n}=30$ interacting via electrostatic and van der Waals pair potentials. Colloids (nanoparticles) are depicted as green (blue) spheres. Red bonds indicate pairs of aggregated particles. To highlight bonds, colloids are slightly reduced in size.

plored the influence of nanoparticles on the structure and stability of colloidal suspensions. In contrast with most previous experimental and modeling work, our study focuses on mixtures of like-charged (mutually repulsive) particles, in which the colloids have sufficiently high valences that repulsive electrostatic interactions usually dominate over attractive van der Waals interactions. While our results for like-charged colloid-nanoparticle mixtures are not directly comparable with experimental observations reported for unlike-charged mixtures [6-10], they qualitatively corroborate previous conclusions that charged nanoparticles can significantly affect the stability of colloidal suspensions.

By computing radial distribution functions and static structure factors, we have shown that addition of charged nanoparticles to a charge-stabilized colloidal suspension can significantly weaken correlations between colloids. Furthermore, with increasing charge of nanoparticles, while colloid-colloid correlations grow weaker, colloidnanoparticle correlations grow stronger. The latter trend is consistent with amplification of the electrostatic coupling with increasing particle charge. The weakening of colloid-colloid correlations can be interpreted as an enhancement of electrostatic screening by charged nanoparticles, as predicted by a theory of effective electrostatic interactions in charged colloid-nanoparticle mixtures [60]. Our results demonstrate that nanoparticleenhanced screening tends to promote irreversible aggregation of weakly charged colloidal particles in a fluid suspension and can induce melting of colloidal crystals.

The accuracy of the coarse-grained model studied here, and the validity of our physical interpretations of the influence of charged nanoparticles on the structure of charge-stabilized colloidal suspensions, may be checked by more extensive simulations of the primitive model, which includes microions explicitly [22-26]. Future studies may also extend our parameter survey by varying particle sizes, charges, and concentrations and exploring oppositely-charged and multicomponent mixtures. The interplay between electrostatic and van der Waals interactions deserves further exploration, especially across the transition from systems dominated by electrostatic interactions to those dominated by excluded-volume interactions. Also important would be analyzing the influence of nanoparticles on thermodynamic properties (e.g., osmotic pressure) and phase transitions, such as freezing/melting. Such an analysis would require consistently incorporating the volume energy and accounting for the density dependence of the effective pair potentials [58]. In the end, our study may shed light on the possibilities of exploiting nanoparticles to tune effective interactions and guide self-assembly of colloidal materials.

\section{ACKNOWLEDGMENTS}

This work was supported by the National Science Foundation under Grant No. DMR-1106331. We thank Dr. Jun Kyung Chung and Dr. Hartmut Löwen for helpful discussions and acknowledge the Center for Computationally Assisted Science and Technology at North Dakota State University for computing resources. 
(North-Holland, Amsterdam, 1991) pp. 763-931.

[2] I. W. Hamley, Introduction to Soft Matter (Wiley, Chichester, 2000).

[3] R. A. L. Jones, Soft Condensed Matter (Oxford, Oxford, 2002).

[4] J. Israelachvili, Intermolecular and Surface Forces (Academic, London, 1992).

[5] D. F. Evans and H. Wennerström, The Colloidal Domain, 2nd ed. (Wiley-VCH, New York, 1999).

[6] V. Tohver, J. E. Smay, A. Braem, P. V. Braun, and J. A. Lewis, PNAS 98, 8950 (2001).

[7] V. Tohver, A. Chan, O. Sakurada, and J. A. Lewis, Langmuir 17, 8414 (2001).

[8] A. T. Chan and J. A. Lewis, Langmuir 21, 8576 (2005).

[9] A. T. Chan and J. A. Lewis, Langmuir 24, 11399 (2008).

[10] F. Zhang, G. G. Long, P. R. Jemian, J. Ilavsky, V. T. Milam, and J. A. Lewis, Langmuir 24, 6504 (2008).

[11] C. J. Martinez, J. Liu, S. K. Rhodes, E. Luijten, E. R. Weeks, and J. A. Lewis, Langmuir 21, 9978 (2005).

[12] J. F. Gilchrist, A. T. Chan, E. R. Weeks, and J. A. Lewis, Langmuir 21, 11040 (2005).

[13] X. Hong and G. A. Willing, Langmuir 25, 4929 (2009).

[14] S. Buzzaccaro, R. Piazza, J. Colombo, and A. Parola, J. Chem. Phys. 132, 124902 (2010).

[15] S. Savarala, S. Ahmed, M. A. Ilies, and S. L. Wunder, ACS Nano 5, 2619 (2011).

[16] X. Xing, G. Sun, Z. Li, and T. Ngai, Langmuir 28, 16022 (2012).

[17] D. Herman and J. Y. Walz, Langmuir 29, 5982 (2013).

[18] D. Herman and J. Y. Walz, Langmuir 31, 4844 (2015).

[19] S. N. Kazi, A. Badarudin, M. N. M. Zubir, H. N. Ming, M. Misran, E. Sadeghinezhad, M. Mehrali, and N. I. Syuhada, Nanoscale Res. Lett. 10, 212 (2015).

[20] D. Herman and J. Y. Walz, J. Coll. Interf. Sci. 449, 143 (2015).

[21] M. N. M. Zubir, A. Badarudin, S. Kazi, M. Misran, A. Amiri, R. Sadri, and S. Khalid, J. Coll. Interf. Sci. 454, 245 (2015).

[22] A. A. Louis, E. Allahyarov, H. Löwen, and R. Roth, Phys. Rev. E 65, 061407 (2002).

[23] J. Liu and E. Luijten, Phys. Rev. Lett. 93, 247802 (2004).

[24] J. Rydén, M. Ullner, and P. Linse, J. Chem. Phys. 123, 034909 (2005).

[25] E. Sanz, C. Valeriani, D. Frenkel, and M. Dijkstra, Phys. Rev. Lett. 99, 055501 (2007).

[26] M. Bier, R. van Roij, and M. Dijkstra, J. Chem. Phys. 133, 124501 (2010).

[27] R. Krause, B. D'Aguanno, J. M. Mendez-Alcaraz, G. Nägele, R. Klein, and R. Weber, J. Phys.: Condens. Matter 3, 4459 (1991).

[28] B. D'Aguanno, R. Krause, J. M. Mendez-Alcaraz, and R. Klein, J. Phys.: Condens. Matter 4, 3077 (1992).

[29] H. Löwen, J.-N. Roux, and J.-P. Hansen, J. Phys.: Condens. Matter 3, 997 (1991).

[30] H. Ruiz-Estrada, M. Medina-Noyola, and G. Nägele, Physica A 168, 919 (1990).

[31] B. D'Aguanno and R. Klein, Phys. Rev. A 46, 7652 (1992).

[32] S. Karanikas and A. A. Louis, Phys. Rev. Lett. 93, 248303 (2004).

[33] E. N. Scheer and K. S. Schweizer, J. Chem. Phys. 128, 164905 (2008).

[34] L. E. Sánchez-Díaz, A. Vizcarra-Rendón, and
M. Medina-Noyola, J. Chem. Phys. 132, 234506 (2010).

[35] L. E. Sánchez-Díaz, G. A. Mendez-Maldonado, M. González-Melchor, H. Ruiz-Estrada, and M. MedinaNoyola, J. Chem. Phys. 135, 14504 (2011).

[36] G. J. Ojeda-Mendoza, A. Moncho Jordá, P. GonzálezMozuelos, C. Haro-Pérez, and L. F. Rojas-Ochoa, Soft Matter 14, 1355 (2018).

[37] A. Torres, A. Cuetos, M. Dijkstra, and R. van Roij, Phys. Rev. E 77, 031402 (2008).

[38] A. Torres, G. Téllez, and R. van Roij, J. Chem. Phys. 128, 154906 (2008).

[39] J. M. Falcón-González and R. Castañeda-Priego, Phys. Rev. E 83, 041401 (2011).

[40] H. Huang and E. Ruckenstein, J. Phys. Chem. B 117, 6318 (2013).

[41] H. Huang and E. Ruckenstein, Colloid Surface A 436, 862 (2013).

[42] K. Barros and E. Luijten, Phys. Rev. Lett. 113, 017801 (2014).

[43] J. K. Chung and A. R. Denton, Phys. Rev. E 88, 022306 (2013).

[44] R. van Roij and J. P. Hansen, Phys. Rev. Lett. 79, 3082 (1997).

[45] A. R. Denton, J. Phys.: Condens. Matter 11, 10061 (1999).

[46] A. R. Denton, Phys. Rev. E 62, 3855 (2000).

[47] A. R. Denton, in Nanostructured Soft Matter: Experiment, Theory, Simulation and Perspectives, edited by A. V. Zvelindovsky (Springer, 2007) pp. 395-433.

[48] E. Allahyarov, H. Löwen, and S. Trigger, Phys. Rev. E 57, 5818 (1998).

[49] E. Allahyarov and H. Löwen, J. Phys.: Condens. Matter 21, 424117 (2009).

[50] A.-P. Hynninen, C. G. Christova, R. van Roij, A. van Blaaderen, and M. Dijkstra, Phys. Rev. Lett. 96, 138308 (2006).

[51] B. V. Derjaguin and L. Landau, Acta Physicochim. URSS 14, 633 (1941).

[52] E. J. W. Verwey and J. T. G. Overbeek, Theory of the Stability of Lyophobic Colloids (Elsevier, Amsterdam, 1948).

[53] For documentation, see http://lammps.sandia.gov.

[54] S. Plimpton, J. Comp. Phys. 117, 1 (1995).

[55] J. P. Hansen and I. R. McDonald, Theory of Simple Liquids, 2nd ed. (Academic, London, 1986).

[56] W. Kob and H. C. Andersen, Phys. Rev. E 52, 4134 (1995).

[57] A. R. Denton, J. Phys.: Condens. Matter 20, 494230 (2008).

[58] A. R. Denton, J. Phys.: Condens. Matter 22, 364108 (2010).

[59] B. Lu and A. R. Denton, Commun. Comput. Phys. 7, 235 (2010).

[60] A. R. Denton, Phys. Rev. E 96, 062610 (2017).

[61] M. Dijkstra, R. van Roij, and R. Evans, Phys. Rev. E 59, 5744 (1999).

[62] B. Götzelmann, R. Roth, S. Dietrich, M. Dijkstra, and R. Evans, Europhys. Lett. 47, 398 (1999).

[63] R. Roth, R. Evans, and S. Dietrich, Phys. Rev. E 62, 5360 (2000).

[64] R. Roth, J. Phys.: Condens. Matter 22, 063102 (2010).

[65] J. P. Hansen and L. Verlet, Phys. Rev. 184, 151 (1969). 